\title{
Properties of Simple Metals Beyond the Local Density Approximation of Density Functional Theory
}

\author{
L. M. ALMEIDA, ${ }^{1,2}$ CARLOS FIOLHAIS, ${ }^{2}$ M. CAUSA ${ }^{3}$ \\ ${ }^{1}$ Department of Physics, University of Aveiro, 3810 Aveiro, Portugal \\ ${ }^{2}$ Department of Physics and Center for Computational Physics, University of Coimbra, \\ 3004-516 Coimbra, Portugal \\ ${ }^{3}$ Department of Science and Advanced Technology, University of the Eastern Piedmont "Amedeo \\ Avogadro," Corso Borsalino 54-15100 Alessandria, Italy
}

Received 11 December 2001; accepted 26 December 2001

DOI 10.1002/qua.10399

\begin{abstract}
We calculated lattice parameters, binding energies, bulk moduli, and phase stabilities of some simple metals: $\mathrm{Li}, \mathrm{Be}, \mathrm{Na}, \mathrm{Mg}$, and $\mathrm{Al}$. Our ab initio all-electron calculations were done within the framework of density functional theory using the Crystal-98 program. The accuracy of different functionals for exchange and correlation energies that go beyond the local density approximation (LDA) was tested. The recent metageneralized gradient approximation proposed from Perdew et al. (Phys Rev Lett $1999,82,2544)$ gives lattice parameters that are better than the LDA values but not always better than the results of generalized gradient approximation. (c) 2002 Wiley Periodicals, Inc. Int J Quantum Chem 91: 224-229, 2003
\end{abstract}

Key words: simple metals; density functional theory; metageneralized gradient approximation; Gaussian-type orbitals; Crystal-98

\section{Introduction}

D ensity functional theory (DFT) [1] has become the prime method for electronic structure calculations. It has gained relevance over other methods for handling the many-electron problem such as Hartree-Fock (HF) or configuration interaction $(\mathrm{CI})$. The reasons are the reduced computing

Correspondence to: L. M. Almeida; e-mail: lma@fis.ua.pt. time and improved accuracy of the results. Nevertheless, hybrid methods that combine exact exchange with approximate correlation are also popular [2].

Since Kohn and Sham [3] proposed the local density approximation (LDA) for exchange and correlation as a practical scheme for implementing DFT, this approach has been used with remarkable success for a variety of systems, in particular in solid-state physics. The LDA exchange and correlation energies only depend on the density. However, 
due to its less satisfactory results for molecules and chemical properties, other density functional approximations for exchange and correlation have been proposed and explored. Despite the availability of various nonempirical functionals, a universal functional that probes accurately for a wide range of systems is still being searched.

Trying to go beyond LDA, Becke [4] proposed an exchange functional that contains a parameter fitted to atomic data. That functional is often used together with the correlation functional developed by Lee et al. [5] on the basis of the Colle-Salvetti correlation energy [6]. Such a combination gives good results for molecules but is not satisfactory for solids or the uniform electron gas [7]. This exchange correlation energy is commonly referred to as BLYP and belongs to the class of generalized gradient approximations (GGA) functionals, which depend not only on the density but also on its gradient.

On the other hand, Perdew and coworkers [8] proposed a series of less empirical density functionals for exchange and correlation that are "controled extrapolations" from the LDA. The simplest one is that of Perdew, Burke, and Ernzerhof (PBE) [9], which also belongs to the class of GGAs. More recently, a new functional was proposed by Perdew, Kurth, Zupan, and Blaha (PKZB) [10] that belongs to the more sophisticated class of metaGGA (MGGA). In MGGA functionals, besides the density and its gradient, the kinetic energy density is used explicitly. Therefore, the MGGA combines usual GGA ideas with the use of orbitals, a concept that is central in implicit functionals like the optimized effective potential (OEP) [11]. The new functional has to be tested and compared with previous ones.

In this article, we report on electronic structure calculations of some simple metals $(\mathrm{Li}, \mathrm{Be}, \mathrm{Na}, \mathrm{Mg}$, and $\mathrm{Al}$ ) using the Crystal-98 program, which employs Gaussian basis sets. We compare the results of LDA, PBE, BLYP, and PKZB functionals for equilibrium lattice parameters, binding energies, and bulk moduli. We also evaluate the relative stability of different cubic lattices.

Many $a b$ initio calculations of structural properties of simple metals have been published. They were done with a wide range of methods and basis sets: plane waves (PWs) [12], linearized augmented plane waves (LAPWs) [7], or Gaussian-type orbitals (GTOs) $[13,14]$. We compare our results with some of them, devoting special attention to the performance of the PKZB functional. The calculated prop- erties are also compared with experimental data at zero temperature or close to it.

\section{Calculations}

MGGA exchange and correlation energies were implemented by us in the program Crystal-98 [15] because this is not a standard feature of that code. MGGA exchange and correlation energies are calculated a posteriori (i.e., after LDA) as the program does with other functionals.

Crystal-98 code is an all-electron program that solves the Kohn-Sham equation of DFT. It uses a basis set made up of GTOs. Bloch functions with the lattice periodicity are constructed from the Gaussian basis set. Then, a linear combination of GTO (LCGTO) is taken.

The use of GTO represents, in fact, an approximation. Thus, the correct choice of the basis set has a major importance. In general, we started from the basis sets already tested in Crystal-98 for the same atoms in different compounds. In the case of $\mathrm{Li}$, we started from the standard 6-21G GTO proposed by Pople and coworkers, which is a feature built into Crystal-98 [15]. Finally, we optimized exponents and parameters of the corresponding outer atomic orbitals taking the conventional equilibrium structure for each metal (bcc for $\mathrm{Li}$ and $\mathrm{Na}$, fcc for $\mathrm{Al}$, and hcp for Be and $\mathrm{Mg}$ ). For this, we used a pattern direct search minimization [16]. This method does not use information on the energy gradient, avoiding therefore the computation of gradients (contrary to the case of the conjugate gradient method). Another advantage is that we always get a minimum and most probably obtain better minima and not just the nearest local one. In our process, we constrained the search for new optimized Gaussians to exponents higher than 0.06 and rejected the cases of linear dependence. All these optimizations were performed within LDA.

In Table I, we show the best parameters found for each metal in the conventional equilibrium structures. The same parameters were used for alternative structures.

For elements of the third row of the periodic table and below, a $d$ shell was included in the basis set because it has a significant influence on the results. The small contributions of extra $s p$ and $d$ shells affect the prediction of the most stable structure, in particular for Li. The extra $d$ shell is necessary to obtain the correct structure stability in MGGA. Figure 1 shows how the contributions of 
TABLE I Initial Basis sets and exponents of reoptimized and added uncontracted GTOs.

\begin{tabular}{|c|c|c|c|c|c|}
\hline & $\mathrm{Li}$ & $\mathrm{Be}$ & $\mathrm{Na}$ & $\mathrm{Mg}$ & $\mathrm{Al}$ \\
\hline Initial GTO & $6-21 G^{a}$ & $5-111 \mathrm{G}^{\mathrm{b}}$ & $8-511 \mathrm{G}^{\mathrm{c}}$ & $8-61 G^{d}$ & $8-511 \mathrm{G}^{\mathrm{e}}$ \\
\hline \multicolumn{6}{|c|}{ Exponents of outer GTOs } \\
\hline$s p$ & 0.08048 & 0.16545 & 0.08344 & 0.11862 & 0.10485 \\
\hline$s p$ & 2.25744 & 1.08480 & 0.47881 & & 0.51623 \\
\hline$s p$ & & 2.98510 & 2.63086 & & 3.94176 \\
\hline$d$ & 0.26966 & & 0.12833 & 0.18020 & 0.44375 \\
\hline
\end{tabular}

a Pople's basis set included in Crystal-98 [15].

b Ref. [19].

c Ref. [20].

${ }^{\mathrm{d}}$ Ref. [21].

e Ref. [22].

extra $s p$ and $d$ shells may be important. Further inclusion of $s p$ or $d$ GTOs is not worthy because the computing time increases unnecessarily.

Electronic structure calculations often find convergence difficulties for metals. To accelerate the
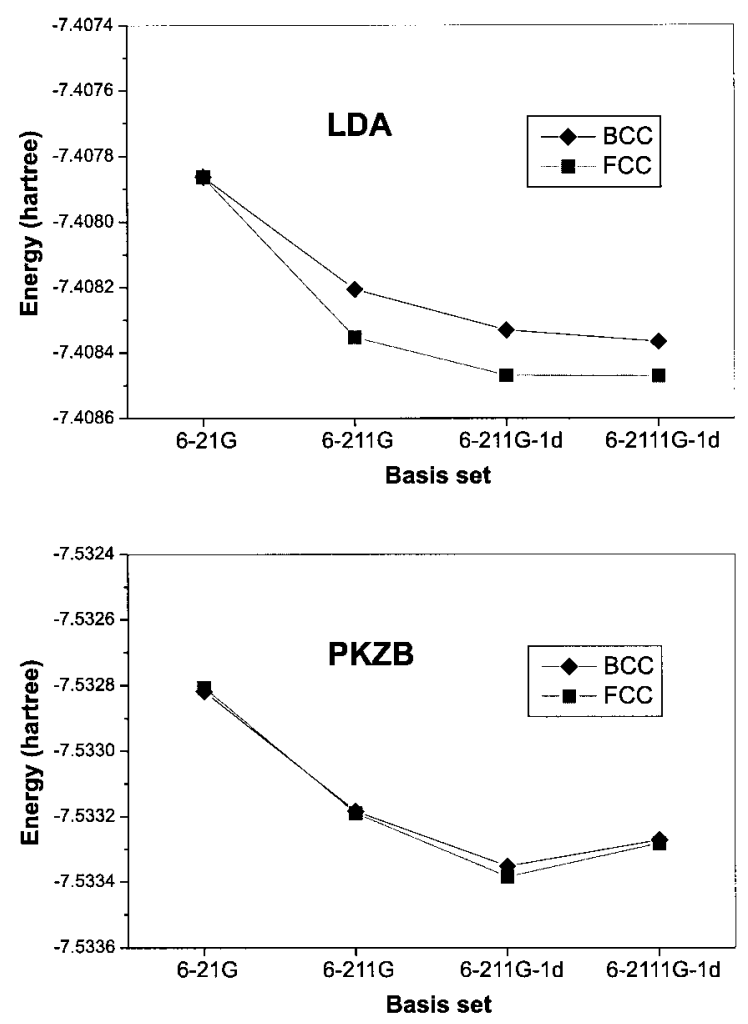

FIGURE 1. Influence of basis set in predicting the most stable structure for Li. In PKZB, the energy difference between fcc and bcc is small, independently of the basis set, but fcc appears below if a $d$ shell is used. self-consistent calculations, we used the Fermi surface smearing technique while finding the best basis set. We introduced a temperature factor in the Fermi distribution function that lies between 0.01 and 0.02 hartree.

The energies given by the various DFT approximations were always evaluated in an a posteriori LDA process. In this way, the initial calculations of the self-consistent Kohn-Sham equations use the exchange and correlation LDA energies and potentials. We took the parametrization of Perdew and Wang 92 [17] for the LDA correlation. Various exchange and correlation functionals were then used taking the LDA density and its gradient, in the case of GGA, and the density, its gradient, and the ki-

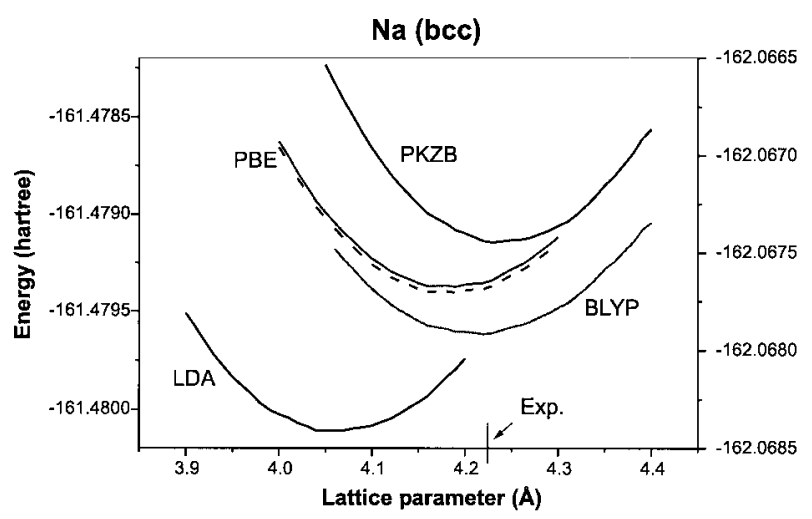

FIGURE 2. Energy vs. lattice parameter for $\mathrm{Na}$ (within LDA, PBE, PKZB, and BLYP). The broken line shows results using $P B E$ densities. All solid lines show results with LDA densities. The vertical scales are correct for LDA and PKZB (on the left and right sides, respectively). The other curves were shifted in energy to fit in the same graph. Exp., experimental lattice parameter. 
PROPERTIES OF SIMPLE METALS BEYOND LDA

TABLE II

Calculated lattice parameters $(\AA \AA)$ and comparison with other works and experimental results.

\begin{tabular}{|c|c|c|c|c|c|c|c|c|c|c|}
\hline & & \multicolumn{4}{|c|}{ Present work } & \multicolumn{4}{|c|}{ Other works } & \multirow[b]{2}{*}{ Exp. } \\
\hline & & LDA & PBE & PKZB & BLYP & LDA & PBE & PKZB & BLYP & \\
\hline \multicolumn{11}{|l|}{$\mathrm{Li}$} \\
\hline bcc & $a$ & 3.37 & 3.45 & 3.52 & 3.42 & $3.41,^{\mathrm{a}} 3.35^{\mathrm{b}}$ & $3.47,{ }^{\mathrm{a}} 3.42^{\mathrm{b}}$ & & $3.47^{\mathrm{a}}$ & $3.48,{ }^{a} 3.49^{b}$ \\
\hline fcc & $a$ & 4.26 & 4.32 & 4.45 & 4.32 & $4.22^{b}$ & $4.31^{\mathrm{b}}$ & & & \\
\hline \multicolumn{11}{|l|}{$\mathrm{Be}$} \\
\hline \multirow[t]{3}{*}{ hcp } & $a$ & 2.22 & 2.25 & 2.28 & 2.25 & $2.25^{\mathrm{c}}$ & & & & $2.29^{c}$ \\
\hline & $c$ & 3.52 & 3.56 & 3.61 & 3.56 & $3.57^{c}$ & & & & $3.58^{c}$ \\
\hline & $c / a$ & 1.58 & 1.58 & 1.58 & 1.58 & $1.59^{\mathrm{c}}$ & & & & $1.57^{\mathrm{c}}$ \\
\hline \multicolumn{11}{|l|}{$\mathrm{Na}$} \\
\hline bcc & $a$ & 4.06 & 4.19 & 4.23 & 4.22 & $4.05^{a, b, d}$ & $4.20,^{\mathrm{a}, \mathrm{d}} 4.19^{\mathrm{b}}$ & $4.32^{d}$ & $4.22,{ }^{a} 4.22^{d}$ & $4.22,^{a} 4.23^{b, d}$ \\
\hline fcc & $a$ & 5.13 & 5.26 & 5.34 & 5.33 & $5.11^{\mathrm{b}}$ & $5.29^{b}$ & & & \\
\hline \multicolumn{11}{|l|}{$\mathrm{Mg}$} \\
\hline \multirow[t]{3}{*}{ hcp } & $a$ & 3.12 & 3.19 & 3.18 & 3.21 & $3.18,,^{e} 3.13^{f}$ & & & & $3.21^{e}$ \\
\hline & $c$ & 5.05 & 5.15 & 5.14 & 5.23 & $5.16,^{e} 5.00^{f}$ & & & & $5.21^{\mathrm{e}}$ \\
\hline & $c / a$ & 1.62 & 1.62 & 1.61 & 1.63 & $1.62,^{e} 1.60^{f}$ & & & & $1.62^{\mathrm{e}}$ \\
\hline \multicolumn{11}{|c|}{ 1.02, 1.00 } \\
\hline bcc & $a$ & 3.16 & 3.23 & 3.19 & 3.30 & $3.22^{\mathrm{g}}$ & & & & \\
\hline fcc & $a$ & 3.97 & 4.01 & 3.99 & 4.07 & $4.02,{ }^{\mathrm{g}} 3.98^{\mathrm{d}}$ & $4.04^{b}$ & $4.02^{d}$ & $4.10^{d}$ & $4.02,,^{g} 4.05^{d}$ \\
\hline $\begin{array}{l}{ }^{\mathrm{a}} \mathrm{LCC} \\
\mathrm{b} P W \\
{ }^{\mathrm{c}} \mathrm{PW} \\
{ }^{\mathrm{c}} \mathrm{PW} \\
{ }^{\mathrm{d}} \mathrm{LAF} \\
{ }^{\mathrm{e}} \mathrm{PW} \\
{ }^{\mathrm{f}} \mathrm{LCG} \\
{ }^{\mathrm{g}} \mathrm{LCC}\end{array}$ & $\begin{array}{l}\text { TTO R } \\
\text { Ref. [ } \\
\text { Ref. [ } \\
\text { W Re } \\
\text { Ref. [ } \\
\text { TO Re } \\
\text { TTO R }\end{array}$ & $\begin{array}{l}{[13] .} \\
] . \\
] . \\
{[7] .} \\
\text { ]. } \\
{[26] .} \\
{[14] .}\end{array}$ & & & & & & & & \\
\hline
\end{tabular}

netic energy density, in the case of MGGA. Total binding energies were evaluated as a function of lattice parameter (see Fig. 2 for Na). The non-selfconsistent method avoids the more expensive calculation of exchange and correlation potentials corresponding to non-LDA functionals. In Crystal-98, only some GGA potentials are implemented, such as PBE. For MGGA, we would even have to resort to implicit methods [11]. We tested the change in the shape of energy-volume curve employing the PBE potential, turning out that the change is small (see Fig. 2). No essential difference in optimal lattice parameters was found. We assumed that the same happens for more sophisticated potentials.

For Li, Na, and Al, for bcc and fcc structures, 145 special $k$ points obtained with the method of Monkhorst and Pack [18] were used in the first Brillouin zone. For Mg and Be, in hcp structure, 192 $k$ points were used. The convergence with respect to the increase of the number of special $k$ points was checked. In all cases, we found energy differences lower than 0.00002 hartree and less than $0.0001 \%$.

\section{Results}

To start, look at the optimal lattice parameters (Table II). The structures chosen were the cubic ones for $\mathrm{Li}$ and $\mathrm{Na}$ because these metals show bcc structure at room temperature (at zero temperature they show a $9 R$ phase [23], with hexagonal symmetry but a big unit cell). For Be and Mg (hcp structure), we calculated the optimal $c / a$ ratio in the different DFT approximations.

We see from Table II that our LDA, PBE, and BLYP results agree in general with published ones. Our new MGGA results improve the energies of previous functionals for $\mathrm{Li}$ and $\mathrm{Na}$ (for $\mathrm{Na}$, there is a small discrepancy with the calculation of Ref. [7], which used the same functional but a different computational technique). A remarkable fact is that all DFT approaches lead to the same $c / a$ ratios for Be and $\mathrm{Mg}$, in both cases close to the experimental ones.

With respect to the bulk moduli (Table III), the results are somewhat scattered in theory as well as 
ALMEIDA, FIOLHAIS, AND CAUSÀ

TABLE III

Calculated bulk moduli (in GPa) and comparison with other works and with experimental results.

\begin{tabular}{|c|c|c|c|c|c|c|c|c|c|c|}
\hline & & \multicolumn{4}{|c|}{ Present work } & \multicolumn{4}{|c|}{ Other works } & \multirow[b]{2}{*}{ Exp. } \\
\hline & & LDA & PBE & PKZB & BLYP & LDA & PBE & PKZB & BLYP & \\
\hline \multirow[t]{2}{*}{$\mathrm{Li}$} & & & & & & & & & & $13.0,^{a}$ \\
\hline & bcc & 15.1 & 14.3 & 14.0 & 14.1 & $14.6,^{\mathrm{a}} 14.9^{\mathrm{b}}$ & $14.5,^{\mathrm{a}} 13.5^{\mathrm{b}}$ & & $13.8^{a}$ & $11.8^{\mathrm{a}}$ \\
\hline $\mathrm{Be}$ & hcp & 133.6 & 129.2 & 127.8 & 124.0 & $131^{\mathrm{c}}$ & & & & $127^{\mathrm{C}}$ \\
\hline $\mathrm{Na}$ & bcc & 9.7 & 8.3 & 11.9 & 9.1 & $9.1,,^{a, b} 9.2^{d}$ & $6.8,^{a} 7.6,^{d} 7.7^{b}$ & $7.0^{d}$ & $5.9,^{a} 7.1^{d}$ & $6.2,^{a} 6.9^{d}$ \\
\hline $\mathrm{Mg}$ & hcp & 43.2 & 41.6 & 52.1 & 41.1 & $44.92^{\mathrm{e}}$ & & & & $36.9^{\mathrm{e}}$ \\
\hline $\mathrm{Al}$ & fcc & 78.4 & 79.7 & 87.6 & 70.2 & $79.7,^{f} 84.0^{d}$ & $77.3^{d}$ & $90.5^{d}$ & $58.6^{d}$ & $72.7,{ }^{f} 77.3^{d}$ \\
\hline
\end{tabular}

${ }^{a}$ LCGTO Ref. [13].

${ }^{b}$ PW Ref. [12].

${ }^{\mathrm{c}}$ PW Ref. [24].

${ }^{\mathrm{d}}$ LAPW Ref. [7].

e LCGTO Ref. [26].

${ }^{f}$ LCGTO Ref. [14].

experimentally. MGGA only improves the bulk modulus of Li, PBE being better for $\mathrm{Na}$ and $\mathrm{Al}$, and BLYP being better for Be and Mg. The bulk moduli were obtained, for cubic structures, by fitting the energy curve to a Taylor expansion around equilibrium lattice values. For hcp structures, we used the Murnaghan equation of state [27].

Table IV shows structural energy differences for $\mathrm{Li}, \mathrm{Na}$, and $\mathrm{Al}$. The most favorable structure is fcc for LDA, PBE, PKZB, and BLYP in all those metals except PKZB for $\mathrm{Na}$, which yields bcc.

\section{Conclusions}

Using Crystal-98, we calculated the energetics of some simple metals using various DFT approximations for exchange and correlation. In particular, we presented new results with the recent MGGA of Perdew et al. [10]. The conclusion is that MGGA improves over GGA in some cases but not always. A more general conclusion arising from the comparison of our DFT results with others is that the GTO basis set seems adequate to describe the struc-

TABLE IV

Energy differences (in mhartree) between bcc and fcc structures.

\begin{tabular}{lrrrr}
\hline & LDA & PBE & PKZB & BLYP \\
\hline $\mathrm{Li}$ & 0.14 & 0.05 & 0.03 & 0.05 \\
$\mathrm{Na}$ & 0.14 & 0.06 & -0.08 & 0.16 \\
$\mathrm{Al}$ & 4.28 & 4.04 & 4.61 & 3.76 \\
\hline
\end{tabular}

tural properties of simple metals if the basis sets are conveniently selected. But, further work remains to be done along these lines.

\section{ACKNOWLEDGMENT}

The authors are grateful to Prof. J. P. Perdew (Tulane University, New Orleans, LA) for his valuable advice.

\section{References}

1. Dreizler, R. M.; Gross, E. K. Density Functional Theory: An Approach to the Quantum Many-Body Problem; SpringerVerlag: Berlin, 1990.

2. Becke, A. D. J Chem Phys 1993, 98, 5648.

3. Kohn, W.; Sham, L. J. Phys Rev A 1965, 140, 1133.

4. Becke, A. D. Phys Rev A 1988, 38, 3098.

5. Lee, C.; Yang, W.; Parr, R. G. Phys Rev B 1988, 37, 785.

6. Colle, R.; Salvetti, D. Theor Chim Acta 1975, 37, 329.

7. Kurth, S.; Perdew, J. P.; Blaha, P. Int J Quantum Chem 1999, 75,889 .

8. Joubert, D., Ed. Density Functionals: Theory and Applications, Lecture Notes in Physics, Vol. 500; Springer-Verlag: Berlin, 1998; p. 8.

9. Perdew, J. P.; Burke, K.; Ernzerhof, M. Phys Rev Lett 1996, 77, 3865.

10. Perdew, J. P.; Kurth, S.; Zupan, A.; Blaha, P. Phys Rev Lett 1999, 82, 2544.

11. Krieger, J. B.; Li, Y.; Iafrate, G. J. Phys Rev A 1992, 46, 5453.

12. Kang, J. H.; Lee, S. H.; Kang, M. H. J Korean Phys Soc 1998, $33,76$.

13. Jaffe, J. E.; Lin, Z.; Hess, A. C. Phys Rev B 1998, 57, 11834.

14. Boettger, J. C.; Trickey, S. B. Phys Rev B 1996, 53, 3007. 


\section{PROPERTIES OF SIMPLE METALS BEYOND LDA}

15. Saunders, N. R.; Dovesi, R.; Roetti, C.; Causà, M.; Harrison, N. M.; Orlando, R.; Zicovich-Wilson, C. M. Crystal-98 User's Manual; University of Torino: Torino, Italy, 1998.

16. Torczon, V. Ph.D. thesis. Rice University: Houston, TX, 1990; Torczon, V. ACM Trans Math Software 1992, 92, 9.

17. Perdew, J. P.; Wang, Y. Phys Rev B 1992, 45, 13244.

18. Monkhorst, H. J.; Pack, J. D. Phys Rev B 1976, 13, 5188.

19. Dovesi, R.; Pisani, C.; Ricca, F.; Roetti, C. Phys Rev B 1982, 25,3731 .

20. Prencipe, M.; Zupan, A.; Dovesi, R.; Aprà, E.; Saunders, V. R. Phys Rev B 1995, 51, 3391.
21. Causà, M.; Dovesi, R.; Pisani, C.; Roetti, C. Phys Rev B 1986, 33, 1308.

22. Catti, M.; Valerio, G.; Dovesi, R.; Causà, M. Phys Rev B 1994, 49, 14179.

23. Blaschko, O.; Dimitriev, V.; Krexner, G.; Toledano, P. Phys Rev 1999, 59, 9095.

24. Chou, M. Y.; Lam, P. K.; Cohen, M. L. Phys Rev B 1983, 28, 4179.

25. Wentzcovitch, R. M.; Cohen, M. L. Phys Rev B 1988, 37, 5571.

26. Baraille, I.; Pouchan, C.; Causà, M.; Marinelli, F. J Phys Condens Matter 1998, 10, 10969.

27. Murnaghan, F. D. Proc Natl Acad Sci USA 1944, 30, 244. 